\title{
Posterior interhemispheric approach and microsurgical resection of a pineal parenchymal neoplasm of intermediate differentiation
}

\author{
Anil Nanda, MD, MPH, Subhas Konar, MD, Piyush Kalakoti, MD, and Tanmoy Maiti, MD \\ Department of Neurosurgery, Louisiana State University Health Sciences Center, Shreveport, Louisiana
}

Owing to a deep-seated location and intricate venous anatomy, pathologies of the posterior third ventricular region pose formidable challenges to the operating neurosurgeon. In this video, we present a case of an elderly Caucasian female with a rare histological variant of a pineal parenchymal mass who presented with gait disturbances and worsening retroorbital headache. Radiological and clinco-histopathological correlates of this rare tumor pathology having intermediate differentiation are highlighted. Briefly outlined are surgical pearls and strategies to minimize complications, as the tumor is approached through the posterior interhemispheric corridor, to achieve a gross-total decompression.

The video can be found here: https://youtu.be/KXwclZ7Ei84.

KEYWORDS occipital interhemispheric; third ventricle; pineal neoplasm; microsurgery; video 